Research Article

\title{
One-Pot Synthesis of Novel Dibenzoxanthenes, Diarylbutanes, and Calix[4]resorcinarenes via Consecutive Pyrrolidine Ring-Closure/Ring-Opening Reactions
}

\author{
Andrey V. Smolobochkin (D, Almir S. Gazizov, Alexander R. Burilov, \\ and Michail A. Pudovik \\ Arbuzov Institute of Organic and Physical Chemistry, FRC Kazan Scientific Center, Russian Academy of Sciences, Kazan, Russia \\ Correspondence should be addressed to Andrey V. Smolobochkin; smolobochkin@iopc.ru
}

Received 17 December 2018; Revised 22 January 2019; Accepted 31 January 2019; Published 15 April 2019

Academic Editor: Sylvain Antoniotti

Copyright (c) 2019 Andrey V. Smolobochkin et al. This is an open access article distributed under the Creative Commons Attribution License, which permits unrestricted use, distribution, and reproduction in any medium, provided the original work is properly cited.

\begin{abstract}
Herein, we report the approach to the otherwise hardly accessible dibenzoxanthenes, diarylbutanes, and calix[4]resorcinarenes possessing urea moieties based on the reaction of $\mathrm{N}$-(4,4-diethoxybutyl)ureas with electron-rich aromatics in strongly acidic media. Unlike the previously developed methods, the proposed approach benefits from one-pot procedure and allows to obtain the target compounds with much higher yields.
\end{abstract}

\section{Introduction}

Diarylmethane derivatives containing two phenolic moieties are of interest due to their wide spectrum biological activity. These compounds are known to inhibit C-C chemokine receptors [1], possess anti-inflammatory [2], antiproliferative [3], antimicrobial [4], anti-HIV [5], and anticancer activity [6]. Some compounds of this class exhibit antibacterial properties $[7,8]$. Additionally, diarylmethane derivatives containing an urea fragment can be used to treat hyperparathyroidism [9-11], malaria [12], and atherosclerosis [13] and inhibit lysine-specific demethylase 1 (LSD1) [14], DNA topoisomerase [15], and epoxy hydrolase [16].

The most general approach to these compounds is the acidic media condensation of electron-rich aromatic nucleophiles with aldehydes [17-19] or acetals [20-22] (Scheme 1(A)). However, this method is inapplicable in case of acetals containing urea moiety, since these compounds are subject to intramolecular cyclization in acidic media [23-26]. Earlier, we have developed the approach to diarylbutanes, dibenzoxanthenes, and calixarenes possessing urea fragment via acid-catalyzed ring opening of 2-(2hydroxynaphthalene-1-yl)pyrrolidine-1-carboxamides
$[27,28]$ (Scheme 1(B)). Although the proposed approach benefits from mild reaction conditions and usage of inexpensive trifluoroacetic acid as catalyst, its certain disadvantage is the necessity of preliminary synthesis of intermediate 2-(2-hydroxynaphthalene-1-yl)pyrrolidines $\mathbf{1}$. At the same time, methods allowing "one-pot" synthesis of the target compounds is of a great importance nowadays due to both their effectiveness and atom economy [29], which are among main principles of green chemistry. Thus, herein we report the improved one-pot approach to the diarylbutane derivatives containing urea moieties starting from easily accessible $N$-(4,4-diethoxybutyl)ureas 2 .

\section{Results and Discussion}

We assumed that pyrrolidine ring opening in strongly acidic media is general for all 2-aryl substituted pyrrolidines. This assumption was supported both by our own observations [27] and synthesis of diphenylbutane derivative upon treatment of $\mathrm{N}$-phenacyl-2-phenylpyrrolidine with triflic acid in benzene solution described by King et al. [30] (Scheme 1(C)). Based on this data, we proposed that carrying out the reaction of $\mathrm{N}$-(4,4-diethoxybutyl)ureas with 

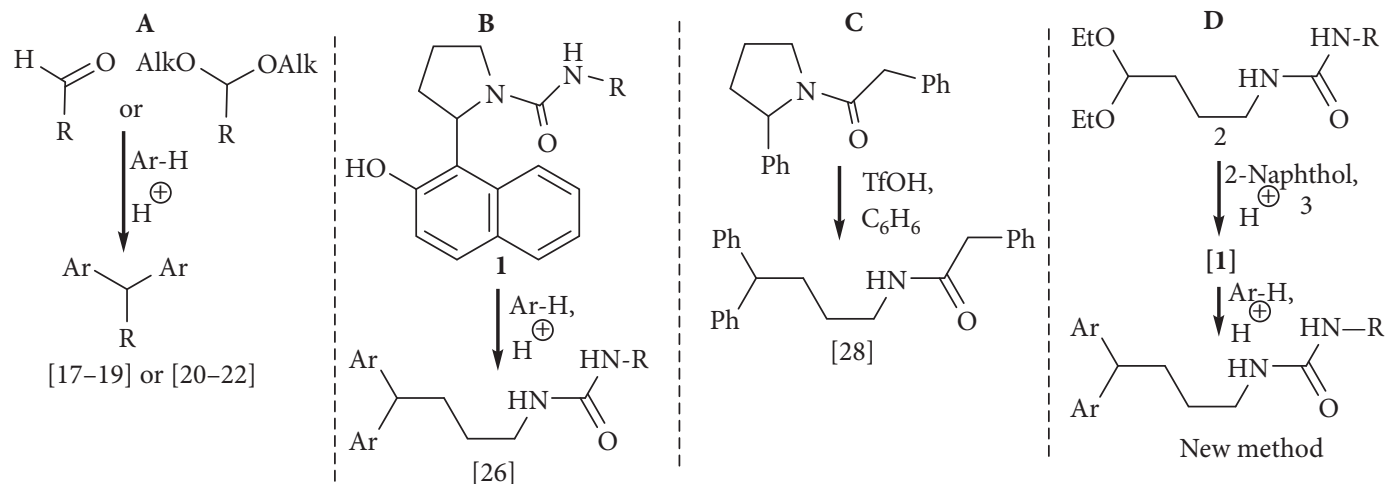

Scheme 1: Synthesis of diarylmethane derivatives.

phenols in strongly acidic media would allow us to obtain appropriate diarylbutanes in one-pot procedure via consecutive pyrrolidine ring closure-ring opening processes. Thus, the need of preliminary synthesis of 2-(2hydroxynaphthalene-1-yl)pyrrolidines would be eliminated.

First, we studied the reaction of ureas $\mathbf{2 a , c}$ with 2naphthol 3. The reaction was carried out in chloroform solution in the presence of 3 -fold excess of trifluoroacetic acid, since these conditions were found to be optimal for the ring opening in 2-(2-hydroxynaphthalene-1-yl)pyrrolidines 1 [27]. However, according to NMR data, in this case the yield of target dibenzoxanthenes 5a,c appeared to be rather low. The main products were previously described by us 2naphthylpyrrolidine derivatives 1a,c [24] (Scheme 2). Next, we gradually increased the amount of trifluoroacetic acid used. Upon increasing the excess of trifluoroacetic acid up to 20 -fold, the reaction led to the formation of target dibenzoxanthenes with about $80 \%$ yield (Table 1, Nos. 1 and 2 ). Naphthalene-2,7-diol 4 reacted under the same conditions with urea $\mathbf{2 b}$ with the formation of previously unknown 2,12-dihydroxydibenzoxanthene derivative $\mathbf{6 b}$ containing urea moiety (Table 1, No. 3).

Further, 4-bromoresorcinol and sesamol were involved into this reaction. The choice of the substrates was based on their known biological activity. For example, sesamol exhibits antioxidant properties $[31,32]$ and is a part of the antidepressant paroxetine structure [33-35] and bromoresorcinol dimers are known for their antimicrobial activity [4]. The reaction of these compounds with ureas $2 \mathbf{a}-\mathbf{c}$ led to the diarylbutane derivatives $7 \mathrm{a}$ and $\mathbf{8 a}-\mathbf{c}$ (Scheme 3, Table 1, Nos. 4-7). Hydroxycoumarine, which is part of the many biologically active compounds [36-39], also successfully undergoes this reaction, leading to the formation of bis(4hydroxy-2H-chromen-2-one) derivative 9a (Table 1, No. 8).

The applicability of this approach to the synthesis of macrocyclic compounds has been demonstrated as well using 2-methylresorcinol as a model substrate. The reaction of this phenol with ureas $\mathbf{2 a} \mathbf{a} \mathbf{d}$ resulted in the appropriate calix[4] resorcinarenes 10a,d formation with up to $70 \%$ yield (Scheme 4, Table 1, No. 9, 10).

As seen from Table 1, using $N$-(4,4-diethoxybutyl)ureas 2 as a starting compounds instead of 2-(2-hydroxynaphthalene1 -yl)pyrrolidines allowed us to increase the yields of the target compounds by $16 \%$ in average. Taking into account the losses of the starting material during the preliminary synthesis of 2(2-hydroxynaphthalene-1-yl)pyrrolidines, the overall gain in yield was more than $20 \%$ (Scheme 5 ).

Taking into consideration previously published data $[30,40]$, we proposed the mechanism of this reaction depicted in Scheme 6. The first stage of the reaction is a protonation of ethoxy group and elimination of ethanol molecule. The oxonium cation $\mathbf{A}$ thus formed may further react with phenol molecule, leading to the 2-arylpyrrolidine derivative B as previously described [24]. Subsequent pyrrolidine ring opening in the presence of excess of trifluoroacetic acid followed by interaction with another phenol molecule via the mechanism similar to that of 2-(2hydroxynaphthalene-1-yl)pyrrolidines [27] results in the formation of target compounds $\mathbf{E}$.

In principle, the other pathway is also possible. It includes the protonation of urea moiety of the oxonium cation A, leading to the dication $\mathbf{F}$. The presence of significant positive charge on the nitrogen atom prevents its intramolecular cyclization, and its further reaction with phenol molecule leads to the acyclic intermediate $\mathbf{H}$. Further reaction of this compound with another phenol molecule via the benzylic cation I also results in the formation of final compounds E.

The experimental data present at the moment does not allow us to unequivocally distinguish between these mechanisms. However, taking into account much higher rate of intramolecular cyclization of $N$-(4,4-diethoxybutyl)ureas 2 compared to their intermolecular interaction with phenols, as well as instability of dicationic species in non-superacidic media, the first pathway seems to be more probable. Additionally, no acyclic intermediates were present in massspectra of the reaction mixture, which may also indicate the preference of the first pathway over second one.

\section{Conclusions}

In conclusion, we have developed one-pot approach to the otherwise hardly accessible dibenzoxanthenes, diarylbutanes, and calix[4]resorcinarenes possessing urea moieties via the reaction of $N$-(4,4-diethoxybutyl)ureas with electron-rich aromatics in strongly acidic media. The 


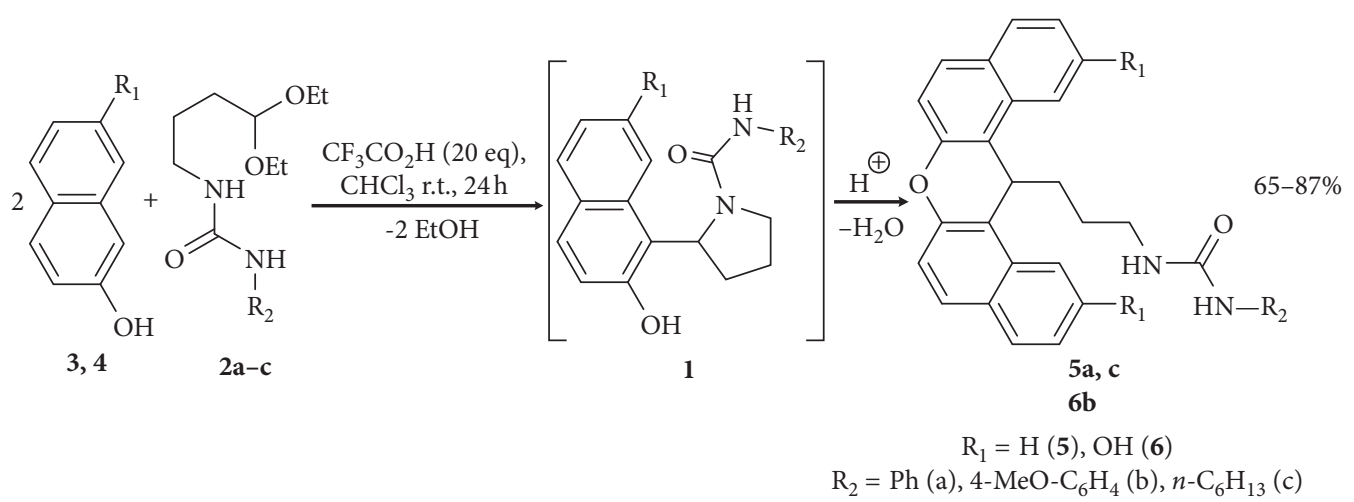

Scheme 2: Synthesis of dibenzoxanthenes.

TABle 1: Synthesis of the compounds 5-10.

\begin{tabular}{lcc}
\hline No. & Compound & Yield $(\%)^{\mathrm{a}, \mathrm{b}}$ \\
\hline 1 & $\mathbf{5 a}$ & $87(78)$ \\
2 & $\mathbf{5 c}$ & $79(53)$ \\
3 & $\mathbf{6 b}$ & $65(57)$ \\
4 & $\mathbf{7 a}$ & $72(28)$ \\
5 & $\mathbf{8 a}$ & $95(74)$ \\
6 & $\mathbf{8 b}$ & $93(69)$ \\
7 & $\mathbf{8 c}$ & $59(48)$ \\
8 & $\mathbf{9 a}$ & $42(60)$ \\
9 & $\mathbf{1 0 a}$ & $70(74)$ \\
10 & $\mathbf{1 0 d}$ & $62(56)$ \\
\hline
\end{tabular}

${ }^{a}$ Isolated yield; ${ }^{b}$ yield from 2-(2-hydroxynaphthalene-1-yl)pyrrolidine according to the procedure described in [27] is given in parentheses.

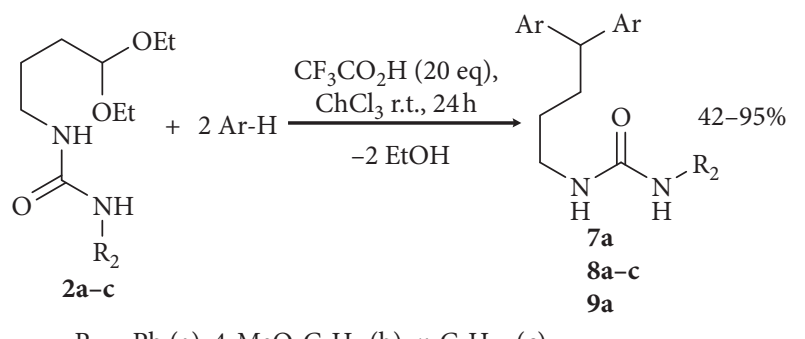

$\mathrm{R}_{2}=\mathrm{Ph}(\mathrm{a}), 4-\mathrm{MeO}-\mathrm{C}_{6} \mathrm{H}_{6}(\mathrm{~b}), n-\mathrm{C}_{6} \mathrm{H}_{13}$ (c)<smiles>CC(C)c1cc2c(cc1O)O[C@H](c1ccc3oc(=O)c(C(C)C)c(O)c3c1)O2</smiles>

Scheme 3: Synthesis of diarylbutanes.

reaction presumably proceeds via consecutive pyrrolidine ring closure-ring opening stages. The proposed approach, in contrast to the previously developed method, does not require an isolation of intermediates and allows to obtain target compounds with much higher yields.

\section{Experimental}

IR spectra were recorded on a UR-20 spectrometer in the $400-3600 \mathrm{~cm}^{-1}$ range in $\mathrm{KBr} .{ }^{1} \mathrm{H}$ NMR spectra were recorded on a Bruker MSL 400 spectrometer $(400 \mathrm{MHz})$ with respect to the signals of residual protons of deuterated solvent $\left(\mathrm{DMSO}-\mathrm{d}_{6}\right) \cdot{ }^{13} \mathrm{C} \mathrm{NMR}$ spectra were recorded on a Bruker Avance $600(150 \mathrm{MHz})$ spectrometer relative to signals of residual protons of deuterated solvent (DMSO- $\left.\mathrm{d}_{6}\right)$. MALDI mass-spectra are obtained on a mass spectrometer UltraFlex III TOF/TOF (Bruker Daltonik GmbH, Bremen, Germany) in a linear mode. The laser is Nd:YAG, $\lambda=266 \mathrm{~nm}$.

The data were processed with the FlexAnalysis 3.0 program (Bruker Daltonik GmbH, Bremen, Germany). Positively charged ions were fixed, and a metal target was used. 2,5-Dihydroxybenzoic acid (DHB) was used as a matrix. Elemental analysis is performed on a Carlo Erba device EA 1108. The melting points are determined in glass capillaries on a Stuart SMP 10 instrument.

2-(2-Hydroxynaphthalen-1-yl)pyrrolidine-1-carboxamides 1a-d were obtained as described previously [24, 25].

4.1. General Method for the Synthesis of Dibenzoxanthenes $5 \mathbf{a}, \mathbf{b}$ and $\mathbf{6 c}$ [27]. To a solution of $1.10 \mathrm{mmol}$ naphthol 4 or 5 in $5 \mathrm{ml}$ of dry chloroform, $0.55 \mathrm{mmol} 2-(2-$ hydroxynaphthalen-1-yl)pyrrolidine-1-carboxamides 1 and $2 \mathrm{ml}$ trifluoroacetic acid were added. The mixture was stirred at room temperature for $72 \mathrm{~h}$. Solvent was evaporated in vacuum. Residue was washed with diethyl ether, filtered, and dried in vacuum ( $1 \mathrm{~h}, 0.01$ Torr) to give the title compound.

4.2. General Method for the Synthesis of Dibenzoxanthenes $5 \mathbf{a}, \mathbf{c}$ and $\mathbf{6 b}$. To a mixture of $1.17 \mathrm{mmol}$ of naphthol, $5 \mathrm{ml}$ of chloroform and $0.59 \mathrm{mmol}$ of acetal 2, and $2 \mathrm{ml}$ of trifluoroacetic acid were added. The reaction mixture was stirred for 24 hours at room temperature, the solvent was removed in vacuum, and the residue was washed with diethyl ether and dried in vacuum.

4.2.1. 1-(3-(14H-Dibenzo[a,j]xanthen-14-yl)pro-pyl)-3-phenylurea (5a). White crystals, m.p. $240^{\circ} \mathrm{C}-241^{\circ} \mathrm{C}$, yield $87 \%$. IR $\left(\mathrm{cm}^{-1}\right.$, $\mathrm{KBr}): 1592,1650,2937,3065,3328 .{ }^{1} \mathrm{H}$ NMR ( $\delta$ ppm, DMSO$\left.d_{6}\right)$ : $1.00-1.11\left(\mathrm{~m}, 2 \mathrm{H}, \mathrm{CH}_{2}\right), 1.89-1.99\left(\mathrm{~m}, 2 \mathrm{H}, \mathrm{CH}_{2}\right)$, 2.71-2.79 (m, 2H, $\left.\mathrm{CH}_{2}\right), 5.72-5.77(\mathrm{~m}, 1 \mathrm{H}, \mathrm{CH}), 5.81-8.84$ $(\mathrm{m}, 1 \mathrm{H}, \mathrm{NH}), 6.82\left(\mathrm{t}, J=7.1 \mathrm{~Hz}, 1 \mathrm{H}, \mathrm{CH}_{\mathrm{Ar}}\right), 7.13(\mathrm{t}, 2 \mathrm{H}$, $\left.J=8.2 \mathrm{~Hz}, \mathrm{CH}_{\mathrm{Ar}}\right), 7.23\left(\mathrm{~d}, 2 \mathrm{H}, J=7.8 \mathrm{~Hz}, \mathrm{CH}_{\mathrm{Ar}}\right), 7.44(\mathrm{~d}, 2 \mathrm{H}$, $\left.J=8.9 \mathrm{~Hz}, \mathrm{CH}_{\mathrm{Ar}}\right), 7.49-7.54\left(\mathrm{~m}, 2 \mathrm{H}, \mathrm{CH}_{\mathrm{Ar}}\right), 7.65-7.71(\mathrm{~m}$, 

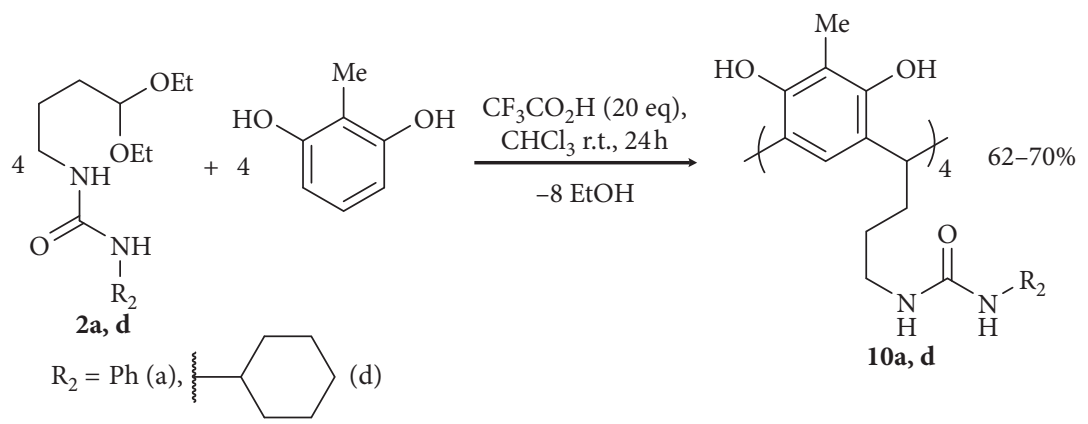

Scheme 4: Synthesis of calixarenes.

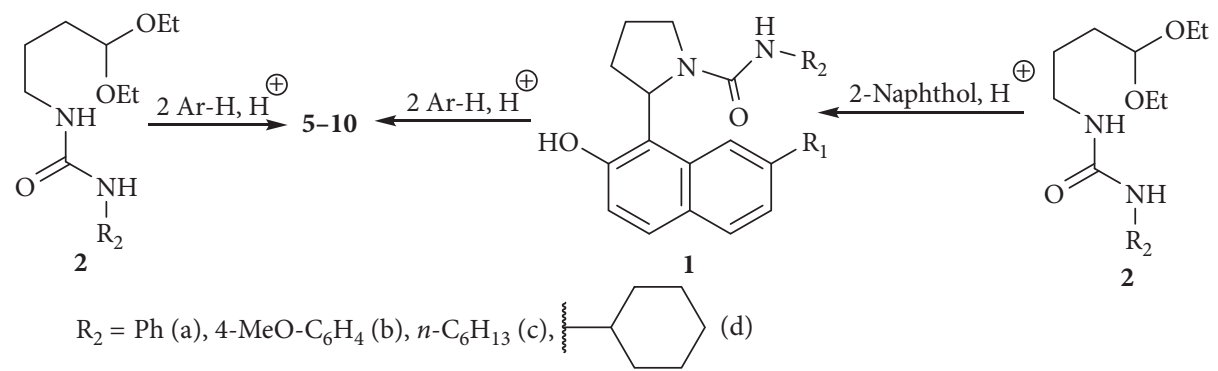

Scheme 5: Synthesis of 2-arylpyrrolidines and polyphenols.

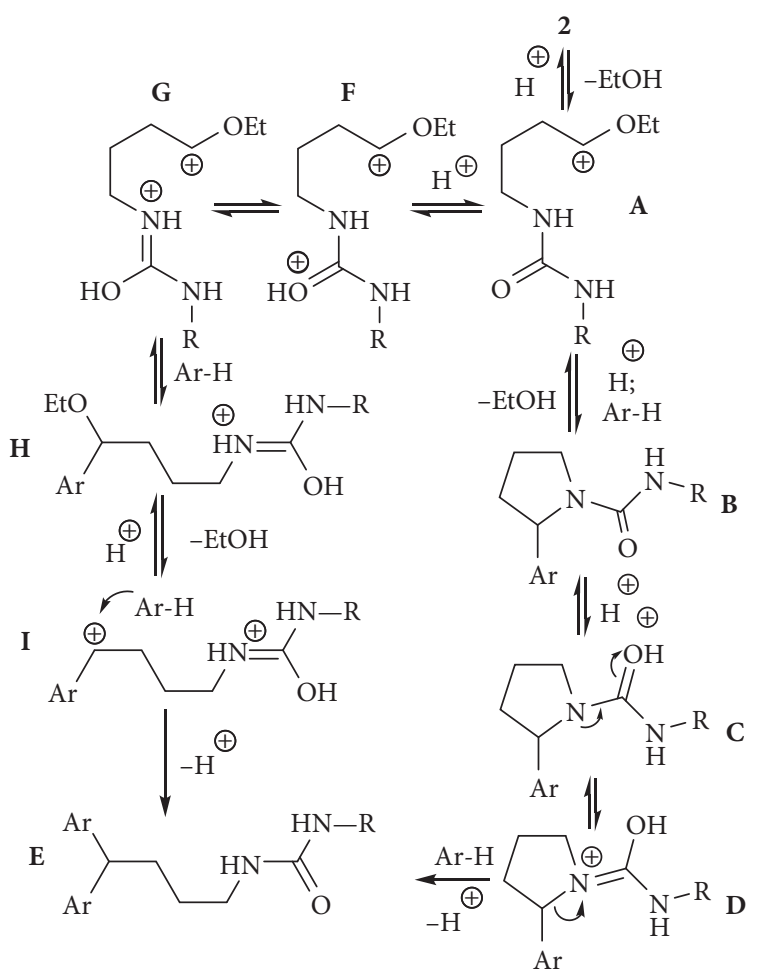

Scheme 6: Proposed mechanism for the substituted ureas formation.

$2 \mathrm{H}, \mathrm{CH}_{\mathrm{Ar}}$ ), $7.91\left(\mathrm{~d}, 2 \mathrm{H}, J=8.9 \mathrm{~Hz}, \mathrm{CH}_{\mathrm{Ar}}\right), 7.96-8.00(\mathrm{~m}, 2 \mathrm{H}$, $\mathrm{CH}_{\mathrm{Ar}}$ ), 8.15 (br.s, $\left.1 \mathrm{H}, \mathrm{NH}\right), 8.54-8.59\left(\mathrm{~m}, 2 \mathrm{H}, \mathrm{CH}_{\mathrm{Ar}}\right) .{ }^{13} \mathrm{C}$ NMR $\left(\delta\right.$ ppm, DMSO- $\left.d_{6}\right): 26.3,30.3,33.9,39.5,117.0,117.6$, $118.0,121.3,123.6,124.9,127.4,129.0,129.1,131.1,131.5$, $140.9,149.9,155.3$. MALDI TOF, $m / z: 481[\mathrm{M}+\mathrm{Na}]^{+}[27]$.
4.2.2. 1-(3-(14H-Dibenzo[a,j]xanthen-14-yl)propyl)-3-hexylurea (5c). White crystals, m.p. $183^{\circ} \mathrm{C}-184^{\circ} \mathrm{C}$, yield $79 \%$. \%. IR $\left(\mathrm{cm}^{-1}, \mathrm{KBr}\right): 1592,1625,2857,2927,3069,3417 .{ }^{1} \mathrm{H}$ NMR $(\delta$ ppm, DMSO- $\left.d_{6}\right): 0.83\left(\mathrm{t}, 3 \mathrm{H}, J=7.04 \mathrm{~Hz}, \mathrm{CH}_{3}\right), 0.91-1.02$ $\left(\mathrm{m}, 2 \mathrm{H}, \mathrm{CH}_{2}\right), 1.08-1.27\left(\mathrm{~m}, 8 \mathrm{H}, \mathrm{CH}_{2}\right), 1.84-1.93(\mathrm{~m}, 2 \mathrm{H}$, $\mathrm{CH}_{2}$ ), 2.59-2.67 (m, 2H, $\left.\mathrm{CH}_{2}\right), 2.76-2.83\left(\mathrm{~m}, 2 \mathrm{H}, \mathrm{CH}_{2}\right)$, 5.42-5.51 (m, 2H, NH), 5.69-5.74 (m, 1H, CH), 7.43 (d, 2H, $\left.J=8.9 \mathrm{~Hz}, \mathrm{CH}_{\mathrm{Ar}}\right), 7.47-7.54\left(\mathrm{~m}, 2 \mathrm{H}, \mathrm{CH}_{\mathrm{Ar}}\right), 7.64-7.70(\mathrm{~m}$, $\left.2 \mathrm{H}, \mathrm{CH}_{\mathrm{Ar}}\right), 7.91\left(\mathrm{~d}, 2 \mathrm{H}, J=8.9 \mathrm{~Hz}, \mathrm{CH}_{\mathrm{Ar}}\right), 7.94-7.99(\mathrm{~m}$, $2 \mathrm{H}, \mathrm{CH}_{\mathrm{Ar}}$ ), 8.51-8.56 (m, $\left.2 \mathrm{H}, \mathrm{CH}_{\mathrm{Ar}}\right) .{ }^{13} \mathrm{C} \mathrm{NMR}(\delta \mathrm{ppm}$, DMSO- $\left.d_{6}\right): 14.3,15.6,22.5,26.4,26.5,26.5,30.3,31.4,33.9$, $39.4,117.0,117.6,123.5,124.8,127.3,128.9,129.1,131.1$, $131.5,149.8,158.2$. MALDI TOF, $m / z: 489[\mathrm{M}+\mathrm{Na}]^{+}[27]$.

4.2.3. 1-(3-(2,12-Dihydroxy-14H-dibenzo[a,j]xanthen-14-yl) propyl)-3-(4-methoxyphenyl)urea (6b). White crystals, m.p. $183-185^{\circ} \mathrm{C}$, yield $65 \%$. IR (cm $\left.{ }^{-1}, \mathrm{KBr}\right): 1596,2835,3195$, 3314. ${ }^{1} \mathrm{H}$ NMR $\left(\delta\right.$ ppm, DMSO- $\left.d_{6}\right): 1.00-1.14\left(\mathrm{~m}, 2 \mathrm{H}, \mathrm{CH}_{2}\right)$, 2.73-2.87 (m, 2H, $\mathrm{CH}_{2}$ ), 3.62-3.71 (m, 2H, $\left.\mathrm{CH}_{2}\right), 3.66(\mathrm{~s}$, $\left.3 \mathrm{H}, \mathrm{CH}_{3}\right), 5.25(\mathrm{t}, J=4.7 \mathrm{~Hz}, 1 \mathrm{H}, \mathrm{CH}), 5.76(\mathrm{t}, J=5.4 \mathrm{~Hz}, 1 \mathrm{H}$, $\mathrm{NH}), 6.74\left(\mathrm{~d}, J=8.9 \mathrm{~Hz}, 2 \mathrm{H}, \mathrm{CH}_{\mathrm{Ar}}\right), 7.08(\mathrm{~d}, J=7.6 \mathrm{~Hz}, 2 \mathrm{H}$, $\mathrm{CH}_{\mathrm{Ar}}$ ), 7.13-7.18 (m, 4H, $\mathrm{CH}_{\mathrm{Ar}}$ ), 7.57 (s, 2H, $\mathrm{CH}_{\mathrm{Ar}}$ ), 7.75 (d, $\left.J=8.7 \mathrm{~Hz}, 2 \mathrm{H}, \mathrm{CH}_{\mathrm{Ar}}\right), 7.80\left(\mathrm{~d}, J=8.7 \mathrm{~Hz}, 2 \mathrm{H}, \mathrm{CH}_{\mathrm{Ar}}\right), 7.89$ (s, $1 \mathrm{H}, \mathrm{NH}), 9.86$ (s, $2 \mathrm{H}, \mathrm{OH}) .{ }^{13} \mathrm{C} \mathrm{NMR}\left(\delta \mathrm{ppm}, \mathrm{DMSO}-d_{6}\right)$ : $14.52,26.43,30.65,32.69,55.56,105.07,114.21,114.25$, $114.96,117.05,119.92,125.56,128.67,130.81,133.18,134.01$, 150.28, 154.32, 155.67, 156.97. MALDI-TOF: $543[\mathrm{M}+\mathrm{Na}]^{+}$. Anal. Calcd.: $\mathrm{C}_{32} \mathrm{H}_{28} \mathrm{~N}_{2} \mathrm{O}_{5}$ (520), C, 73.83; H, 5.42; N, 5.38. Found: C, 73.98; H, 5.60; N, 5.48.

4.3. General Method for the Synthesis of Diarylbutanes 7a, 8a-c, 9a [27]. To a mixture of $0.30 \mathrm{mmol}$ pyrrolidine-1- 
carboxamide $\mathbf{1}$ in $5 \mathrm{ml}$ of dry chloroform, appropriate $0.90 \mathrm{mmol}$ phenol and $2 \mathrm{ml}$ trifluoroacetic acid were added. The mixture was stirred at room temperature for $72 \mathrm{~h}$. Solvent was evaporated in vacuum. Residue was washed with diethyl ether, filtered, and dried in vacuum ( $1 \mathrm{~h}, 0.01$ Torr) to give the title compound $7 \mathrm{a}, \mathbf{8 a}-\mathbf{c}, \mathbf{9 a}$.

4.4. General Method for the Synthesis of Diarylbutanes 7a, 8a-c, 9a. To a mixture of $1.82 \mathrm{mmol}$ of phenol, $5 \mathrm{ml}$ of chloroform, and $0.91 \mathrm{mmol}$ of acetal 2, $2 \mathrm{ml}$ of trifluoroacetic acid was added. The reaction mixture was stirred for 24 hours at room temperature, the solvent was removed in vacuum, and the residue was washed with diethyl ether and dried in vacuum.

4.4.1. 1-(4,4-Bis(5-bromo-2,4-dihydroxyphenyl)butyl)-3-phenylurea (7a). White crystals, m.p. $132^{\circ} \mathrm{C}-133^{\circ} \mathrm{C}$, yield $72 \%$. IR $\left(\mathrm{cm}^{-1}\right.$, $\mathrm{KBr}): 1597,1652,2868,2937,3402 .{ }^{1} \mathrm{H}$ NMR $(\delta \mathrm{ppm}$, DMSO- $\left.d_{6}\right): 1.27-1.37\left(\mathrm{~m}, 2 \mathrm{H}, \mathrm{CH}_{2}\right), 1.77-1.86(\mathrm{~m}, 2 \mathrm{H}$, $\left.\mathrm{CH}_{2}\right), 3.02-3.10\left(\mathrm{~m}, 2 \mathrm{H}, \mathrm{CH}_{2}\right), 4.25(\mathrm{t}, 1 \mathrm{H}, J=7.9 \mathrm{~Hz}$, $\left.\mathrm{CH}_{\mathrm{Ar}}\right), 6.08(\mathrm{t}, 1 \mathrm{H}, J=5.8 \mathrm{~Hz}, \mathrm{NH}), 6.45\left(\mathrm{~s}, 2 \mathrm{H}, \mathrm{CH}_{\mathrm{Ar}}\right)$, 6.84-6.89 (m, $\left.1 \mathrm{H}, \mathrm{CH}_{\mathrm{Ar}}\right), 7.03\left(\mathrm{~s}, 2 \mathrm{H}, \mathrm{CH}_{\mathrm{Ar}}\right), 7.16-7.22(\mathrm{~m}$, $\left.2 \mathrm{H}, \mathrm{CH}_{\mathrm{Ar}}\right), 7.33-7.38\left(\mathrm{~m}, 2 \mathrm{H}, \mathrm{CH}_{\mathrm{Ar}}\right) .{ }^{13} \mathrm{C} \mathrm{NMR}(\delta \mathrm{ppm}$, DMSO- $\left.d_{6}\right): 29.0,31.5,36.1,65.4,98.2,104.0,118.1,121.3$, $124.4,129.0,131.7,141.0,152.7,155.5,155.6$. MALDI TOF, $m / z: 589[\mathrm{M}+\mathrm{Na}]^{+}[27]$.

4.4.2. 1-(4,4-Bis(6-hydroxybenzo[d][1,3]dioxol-5-yl)butyl)-3phenylurea (8a). White crystals, m.p. $165-166^{\circ} \mathrm{C}$, yield $95 \%$. IR $\left(\mathrm{cm}^{-1}, \mathrm{KBr}\right): 1596,2935,3291,3383 .{ }^{1} \mathrm{H}$ NMR $(\delta \mathrm{ppm}$, DMSO- $\left.d_{6}\right): 1.29-1.39\left(\mathrm{~m}, 2 \mathrm{H}, \mathrm{CH}_{2}\right), 1.80-1.89(\mathrm{~m}, 2 \mathrm{H}$, $\left.\mathrm{CH}_{2}\right), 3.02-3.10\left(\mathrm{~m}, 2 \mathrm{H}, \mathrm{CH}_{2}\right), 4.43(\mathrm{t}, J=7.8 \mathrm{~Hz}, 1 \mathrm{H}, \mathrm{CH})$, $5.83\left(\mathrm{~d}, J=8.3 \mathrm{~Hz}, 4 \mathrm{H}, \mathrm{CH}_{2}\right), 6.07(\mathrm{~s}, 1 \mathrm{H}, \mathrm{NH}), 6.38(\mathrm{~s}, 2 \mathrm{H}$, $\left.\mathrm{CH}_{\mathrm{Ar}}\right), 6.69\left(\mathrm{~s}, 2 \mathrm{H}, \mathrm{CH}_{\mathrm{Ar}}\right), 7.20\left(\mathrm{t}, J=7.6 \mathrm{~Hz}, 2 \mathrm{H}, \mathrm{CH}_{\mathrm{Ar}}\right.$ ), $7.35\left(\mathrm{~d}, J=7.6 \mathrm{~Hz}, 2 \mathrm{H}, \mathrm{CH}_{\mathrm{Ar}}\right), 8.29(\mathrm{~s}, 1 \mathrm{H}, \mathrm{NH}), 8.90(\mathrm{~s}, 2 \mathrm{H}$, $\mathrm{OH}) .{ }^{13} \mathrm{C}$ NMR $\left(\delta\right.$ ppm, DMSO- $\left.d_{6}\right): 15.65,29.08,31.73$, $36.18,98.01,100.80,108.05,118.09,121.36,123.42,129.05$, 140.11, 141.06, 145.47, 149.56, 155.65. MALDI-TOF: 487 $[\mathrm{M}+\mathrm{Na}]^{+}, 503[\mathrm{M}+\mathrm{K}]^{+}$. Anal. Calcd.: $\mathrm{C}_{25} \mathrm{H}_{24} \mathrm{~N}_{2} \mathrm{O}_{7}$ (464), 64.65; H, 5.21; N, 6.03. Found: 64.79; H, 5.11; N, 5.98.

4.4.3. 1-(4,4-Bis(6-hydroxybenzo[d] [1,3]dioxol-5-yl)butyl)-3(4-methoxyphenyl)urea (8b). White crystals, m.p. 130$131^{\circ} \mathrm{C}$, yield $93 \%$. IR $\left(\mathrm{cm}^{-1}, \mathrm{KBr}\right): 1595,2818,3212,3337 .{ }^{1} \mathrm{H}$ NMR $\left(\delta \mathrm{ppm}, \mathrm{DMSO}-d_{6}\right): 1.29-1.37\left(\mathrm{~m}, 2 \mathrm{H}, \mathrm{CH}_{2}\right), 1.79-$ $1.86\left(\mathrm{~m}, 2 \mathrm{H}, \mathrm{CH}_{2}\right), 2.98-3.08\left(\mathrm{~m}, 2 \mathrm{H}, \mathrm{CH}_{2}\right), 3.68(\mathrm{~s}, 3 \mathrm{H}$, $\left.\mathrm{CH}_{3}\right), 4.42(\mathrm{t}, J=7.8 \mathrm{~Hz}, 1 \mathrm{H}, \mathrm{CH}), 5.83(\mathrm{~d}, J=9.5 \mathrm{~Hz}, 4 \mathrm{H}$, $\mathrm{CH}_{2}$ ), 5.95 (s, $\left.1 \mathrm{H}, \mathrm{NH}\right), 6.37\left(\mathrm{~s}, 2 \mathrm{H}, \mathrm{CH}_{\mathrm{Ar}}\right), 6.68(\mathrm{~s}, 2 \mathrm{H}$, $\left.\mathrm{CH}_{\mathrm{Ar}}\right), 6.79\left(\mathrm{~d}, J=9.1 \mathrm{~Hz}, 2 \mathrm{H}, \mathrm{CH}_{\mathrm{Ar}}\right), 7.24(\mathrm{~d}, J=9.1 \mathrm{~Hz}, 2 \mathrm{H}$, $\left.\mathrm{CH}_{\mathrm{Ar}}\right), 8.08\left(\mathrm{~s}, 2 \mathrm{H}, \mathrm{CH}_{\mathrm{Ar}}\right), 8.90(\mathrm{~s}, 2 \mathrm{H}, \mathrm{OH}) .{ }^{13} \mathrm{C} \mathrm{NMR}(\delta$ ppm, DMSO- $\left.d_{6}\right): 29.15,31.73,36.14,55.61,55.68,98.00$, $100.80,108.07,114.38,119.89,123.43,134.20,140.10,145.46$, 149.55, 154.34, 155.90. MALDI-TOF: $507[\mathrm{M}+\mathrm{Na}]^{+}$. Anal. Calcd.: $\mathrm{C}_{26} \mathrm{H}_{26} \mathrm{~N}_{2} \mathrm{O}_{8}$ (494), C, 63.15; H, 5.30; N, 5.67. Found: C, 63.27; H, 5.41; N, 5.85 .
4.4.4. 1-(4,4-Bis(6-hydroxybenzo[d][1,3]dioxol-5-yl)butyl)-3hexylurea (8c). White crystals, m.p. $102-103^{\circ} \mathrm{C}$, yield $59 \%$. IR $\left(\mathrm{cm}^{-1}, \mathrm{KBr}\right): 1597,2719,3147,3214,3362 .{ }^{1} \mathrm{H}$ NMR $(\delta$ ppm, DMSO- $\left.d_{6}\right): 0.84\left(\mathrm{t}, \mathrm{J}=6.4 \mathrm{~Hz}, 3 \mathrm{H}, \mathrm{CH}_{3}\right), 1.21-1.27(\mathrm{~m}$, $\left.8 \mathrm{H}, \mathrm{CH}_{2}\right), 1.31-1.35\left(\mathrm{~m}, 2 \mathrm{H}, \mathrm{CH}_{2}\right), 1.72-1.85\left(\mathrm{~m}, 2 \mathrm{H}, \mathrm{CH}_{2}\right)$, 2.93-2.99 (m, 2H, $\left.\mathrm{CH}_{2}\right), 4.42(\mathrm{t}, J=7.8 \mathrm{~Hz}, 1 \mathrm{H}, \mathrm{CH}), 5.83(\mathrm{~d}$, $\left.J=10.8 \mathrm{~Hz}, 4 \mathrm{H}, \mathrm{CH}_{2}\right), 6.39\left(\mathrm{~s}, 2 \mathrm{H}, \mathrm{CH}_{\mathrm{Ar}}\right), 6.67(\mathrm{~s}, 2 \mathrm{H}$, $\left.\mathrm{CH}_{\mathrm{Ar}}\right), 7.67(\mathrm{~s}, 2 \mathrm{H}, \mathrm{OH}) .{ }^{13} \mathrm{C}$ NMR $\left(\delta \mathrm{ppm}, \mathrm{DMSO}-d_{6}\right)$ : $14.29,22.53,26.51,29.31,30.43,31.50,31.70,36.14,46.68$, 55.21, 98.03, 100.76, 107.96, 119.74, 140.11, 149.54, 153.03, 158.70. MALDI-TOF: $495[\mathrm{M}+\mathrm{Na}]^{+}$. Anal. Calcd.: $\mathrm{C}_{25} \mathrm{H}_{32} \mathrm{~N}_{2} \mathrm{O}_{7}$ (472), C, 63.55; H, 6.83; N, 5.93. Found: C, 63.63; H, 6.99; N, 6.16 .

4.4.5. 1-(4,4-Bis(4-hydroxy-2-oxo-2H-chromen-3-yl)butyl)-3phenylurea (9a). White crystals, m.p. $235-237^{\circ} \mathrm{C}$, yield $42 \%$. IR $\left(\mathrm{cm}^{-1}, \mathrm{KBr}\right): 1595,2810,3198,3358,3334 .{ }^{1} \mathrm{H}$ NMR $(\delta$ ppm, DMSO- $\left.d_{6}\right): 1.28-1.45\left(\mathrm{~m}, 2 \mathrm{H}, \mathrm{CH}_{2}\right), 2.06-2.20(\mathrm{~m}, 2 \mathrm{H}$, $\left.\mathrm{CH}_{2}\right), 2.95-3.10\left(\mathrm{~m}, 2 \mathrm{H}, \mathrm{CH}_{2}\right), 4.89(\mathrm{t}, J=8.3 \mathrm{~Hz}, 1 \mathrm{H}, \mathrm{CH})$, $6.84\left(\mathrm{t}, J=7.5 \mathrm{~Hz}, 1 \mathrm{H}, \mathrm{CH}_{\mathrm{Ar}}\right), 7.14-7.19\left(\mathrm{~m}, 2 \mathrm{H}, \mathrm{CH}_{\mathrm{Ar}}\right)$, 7.25-7.30 (m, $\left.3 \mathrm{H}, \mathrm{CH}_{\mathrm{Ar}}\right), 7.32\left(\mathrm{~d}, J=8.6 \mathrm{~Hz}, 1 \mathrm{H}, \mathrm{CH}_{\mathrm{Ar}}\right)$, 7.34-7.38 (m, 2H, $\left.\mathrm{CH}_{\mathrm{Ar}}\right), 7.53\left(\mathrm{t}, J=6.9 \mathrm{~Hz}, 1 \mathrm{H}, \mathrm{CH}_{\mathrm{Ar}}\right), 7.64$ (t, $J=7.6 \mathrm{~Hz}, 1 \mathrm{H}, \mathrm{NH}), 7.82\left(\mathrm{~d}, J=8.0 \mathrm{~Hz}, 1 \mathrm{H}, \mathrm{CH}_{\mathrm{Ar}}\right), 7.91$ $\left(\mathrm{d}, J=6.7 \mathrm{~Hz}, 2 \mathrm{H}, \mathrm{CH}_{\mathrm{Ar}}\right), 8.27(\mathrm{~s}, 1 \mathrm{H}, \mathrm{NH}) .{ }^{13} \mathrm{C}$ NMR $(\delta$ ppm, DMSO- $d_{6}$ ): $27.57,29.25,32.16,61.66,116.84,118.07$, $123.68,124.26,124.39,129.02,131.79,133.17,141.05,152.58$, 155.60, 162.34, 165.10, 166.10. MALDI-TOF: $513[\mathrm{M}+\mathrm{H}]^{+}$. Anal. Calcd.: $\mathrm{C}_{29} \mathrm{H}_{24} \mathrm{~N}_{2} \mathrm{O}_{7}$ (512), C, 67.96; H, 4.72; N, 5.47. Found: C, 68.11; H, 4.90; N, 5.59.

4.5. General Method for the Synthesis of Calix [4] resorcinarenes 10a,d [27, 41]. To a mixture of $1.25 \mathrm{mmol}$ pyrrolidine-1carboxamide 1 and appropriate $0.16 \mathrm{~g}(1.25 \mathrm{mmol}) 2$ methylresorcinol in $5 \mathrm{ml}$ dry chloroform, $2 \mathrm{ml}$ trifluoroacetic acid was added. The mixture was stirred at room temperature for $72 \mathrm{~h}$. Solvent was evaporated in vacuum. Residue was washed with diethyl ether and acetone, filtered, and dried in vacuum ( $1 \mathrm{~h}, 0.01$ Torr) to give the title compound 10.

4.6. General Methodfor the Synthesis of Calix[4]resorcinarenes $10 \mathrm{a}, \mathbf{d}$. To a mixture of $0.16 \mathrm{~g}(1.25 \mathrm{mmol})$ of 2 -methylresorcinol, $5 \mathrm{ml}$ of chloroform, and $1.25 \mathrm{mmol}$ of acetal 2 , $2 \mathrm{ml}$ of trifluoroacetic acid was added. The reaction mixture was stirred for 24 hours at room temperature, the solvent was removed in vacuum, and the residue was washed with diethyl ether and dried in vacuum.

4.6.1. $1,1^{\prime}, 1^{\prime \prime}, 1^{\prime \prime \prime}-\left(\left(1^{4}, 1^{6}, 3^{4}, 3^{6}, 5^{4}, 5^{6}, 7^{4}, 7^{6}-\right.\right.$ Octahydroxy- $1^{5}, 3^{5}, 5^{5}, 7^{5}-$ tetramethyl-1,3,5,7(1,3)-tetrabenzenacyclooctaphane-2,4,6,8tetrayl)tetrakis(propane-3,1-diyl))tetrakis(3-phenylurea) (10a). White crystals, m.p. $>250^{\circ} \mathrm{C}$, yield $70 \%$. IR $\left(\mathrm{cm}^{-1}, \mathrm{KBr}\right)$ : $1598,1653,2862,2933,3057,3387 .{ }^{1} \mathrm{H}$ NMR $(\delta \mathrm{ppm}$, DMSO- $\left.d_{6}\right): 1.43-1.59\left(\mathrm{~m}, 8 \mathrm{H}, \mathrm{CH}_{2}\right), 2.00-2.18(\mathrm{~m}, 8 \mathrm{H}$, $\left.\mathrm{CH}_{2}\right), 2.04\left(\mathrm{~s}, 12 \mathrm{H}, \mathrm{CH}_{3}\right), 2.23-2.32\left(\mathrm{~m}, 8 \mathrm{H}, \mathrm{CH}_{2}\right), 3.18-$ $3.28\left(\mathrm{~m}, 8 \mathrm{H}, \mathrm{CH}_{2}\right), 4.40(\mathrm{t}, 4 \mathrm{H}, J=7.80 \mathrm{~Hz}, \mathrm{CH}), 6.90-6.96$ $\left(\mathrm{m}, 4 \mathrm{H}, \mathrm{CH}_{\mathrm{Ar}}\right), 7.15\left(\mathrm{~s}, 4 \mathrm{H}, \mathrm{CH}_{\mathrm{Ar}}\right), 7.17-7.22(\mathrm{~m}, 8 \mathrm{H}$, 
$\left.\mathrm{CH}_{\mathrm{Ar}}\right), 7.27-7.34\left(\mathrm{~m}, 8 \mathrm{H}, \mathrm{CH}_{\mathrm{Ar}}\right) .{ }^{13} \mathrm{C}$ NMR $(\delta \mathrm{ppm}, \mathrm{DMSO}-$ $\left.d_{6}\right): 8.5,28.9,31.4,34.3,39.4,112.4,119.2,120.0,122.2$, $124.8,128.5,139.4,149.5,157.2$. MALDI TOF, $m / z: 1249$ $[\mathrm{M}]^{+} ; 1250[\mathrm{M}+\mathrm{H}]^{+} ; 1272[\mathrm{M}+\mathrm{Na}]^{+} ; 1288[\mathrm{M}+\mathrm{K}]^{+}[27]$.

4.6.2. $1,1^{\prime}, 1^{\prime \prime}, 1^{\prime \prime \prime}-((14,16,34,36,54,56,74,76-$ Octahydroxy-15,35,55, 75-tetramethyl-1,3,5,7(1,3)-tetrabenzenacyclooctaphane-2,4,6,8tetrayl)tetrakis(propane-3,1-diyl))tetrakis(3-cyclohexylurea) (10d). White crystals, m.p. $>250^{\circ} \mathrm{C}$, yield $62 \%$. IR $\left(\mathrm{cm}^{-1}, \mathrm{KBr}\right)$ : $1642,2860,3096,3198 .{ }^{1} \mathrm{H}$ NMR ( $\delta \mathrm{ppm}$, DMSO- $\left.d_{6}\right)$ : 1.00-1.17 (m, 12H, $\left.\mathrm{CH}_{2}\right), 1.19-1.36\left(\mathrm{~m}, 12 \mathrm{H}, \mathrm{CH}_{2}\right)$, 1.47-1.55 (m, $\left.4 \mathrm{H}, \mathrm{CH}_{2}\right), 1.58-1.67\left(\mathrm{~m}, 8 \mathrm{H}, \mathrm{CH}_{2}\right), 1.69-1.80$ $\left(\mathrm{m}, 8 \mathrm{H}, \mathrm{CH}_{2}\right), 1.95\left(\mathrm{~s}, 12 \mathrm{H}, \mathrm{CH}_{3}\right), 2.17-2.29(\mathrm{~m}, 4 \mathrm{H}$, $\left.\mathrm{CH}_{2}\right), 2.96-3.10(\mathrm{~m}, 4 \mathrm{H}, \mathrm{CH}), 3.29-3.47\left(\mathrm{~m}, 8 \mathrm{H}, \mathrm{CH}_{2}\right)$, 4.16-4.24 (m, $4 \mathrm{H}, \mathrm{CH}), 5.68(\mathrm{~s}, 4 \mathrm{H}, \mathrm{NH}), 5.79(\mathrm{~s}, 4 \mathrm{H}, \mathrm{NH})$, $7.23\left(\mathrm{~s}, 4 \mathrm{H}, \mathrm{CH}_{\mathrm{Ar}}\right) .{ }^{13} \mathrm{C}$ NMR $\left(\delta \mathrm{ppm}, \mathrm{DMSO}-d_{6}\right): 10.46$, $24.98,25.78,29.47,30.78,33.83,34.51,38.84,48.15,112.18$, $121.45,125.05,149.58,157.96$. MALDI TOF, $m / z: 1273$ $[\mathrm{M}+\mathrm{H}]^{+} ; 1295[\mathrm{M}+\mathrm{Na}]^{+}[41]$.

\section{Data Availability}

The NMR source data used to support the findings of this study are available from the corresponding author upon request.

\section{Conflicts of Interest}

The authors declare that they have no conflicts of interest.

\section{Acknowledgments}

The authors are grateful to the Assigned Spectral-Analytical Center of FRC Kazan Scientific Center of RAS for technical assistance in research.

\section{Supplementary Materials}

Copies of NMR spectra for all of the new compounds. (Supplementary Materials)

\section{References}

[1] M. Imai, T. Shiota, K.-i. Kataoka et al., "Small molecule inhibitors of the CCR2b receptor. Part 1: discovery and optimization of homopiperazine derivatives," Bioorganic \& Medicinal Chemistry Letters, vol. 14, no. 21, pp. 5407-5411, 2004.

[2] S. Cardinal, P.-A. Paquet-Côté, J. Azelmat, C. Bouchard, D. Grenier, and N. Voyer, "Synthesis and anti-inflammatory activity of isoquebecol," Bioorganic \& Medicinal Chemistry, vol. 25, no. 7, pp. 2043-2056, 2017.

[3] K. Pericherla, A. N. Shirazi, V. Kameshwara Rao et al., "Synthesis and antiproliferative activities of quebecol and its analogs," Bioorganic \& Medicinal Chemistry Letters, vol. 23, no. 19 , pp. 5329-5331, 2013.

[4] E. Bouthenet, K.-B. Oh, S. Park, N. K. Nagi, H.-S. Lee, and S. E. Matthews, "Synthesis and antimicrobial activity of brominated resorcinol dimers," Bioorganic \& Medicinal Chemistry Letters, vol. 21, no. 23, pp. 7142-7145, 2011.
[5] S. K. Chauthe, S. B. Bharate, S. Sabde, D. Mitra, K. K. Bhutani, and I. P. Singh, "Biomimetic synthesis and anti-HIV activity of dimeric phloroglucinols," Bioorganic \& Medicinal Chemistry, vol. 18, no. 5, pp. 2029-2036, 2010.

[6] S. K. Chauthe, S. B. Bharate, G. Periyasamy et al., "One pot synthesis and anticancer activity of dimeric phloroglucinols," Bioorganic \& Medicinal Chemistry Letters, vol. 22, no. 6, pp. 2251-2256, 2012.

[7] K. Sumoto, N. Mibu, K. Yokomizo, and M. Uyeda, "Synthesis of 2,2'-dihydroxybisphenols and antiviral activity of some bisphenol derivatives," Chemical \& Pharmaceutical Bulletin, vol. 50, no. 2, pp. 298-300, 2002.

[8] N. Mibu, K. Yokomizo, T. Miyata, and K. Sumoto, "Synthesis and antiviral activities of some heteroaryl-substituted triarylmethanes," Journal of Heterocyclic Chemistry, vol. 47, no. 6, pp. 1434-1438, 2010.

[9] T. Temal, H. Jary, M. Auberval et al., "New potent calcimimetics: I. Discovery of a series of novel trisubstituted ureas," Bioorganic \& Medicinal Chemistry Letters, vol. 23, no. 8, pp. 2451-2454, 2013.

[10] P. Deprez, T. Temal, H. Jary et al., "New potent calcimimetics: II. Discovery of benzothiazole trisubstituted ureas," Bioorganic \& Medicinal Chemistry Letters, vol. 23, no. 8, pp. 2455-2459, 2013.

[11] P. M. Vevert, P. E. Harrington, T. J. Carlson et al., "Metabolism-guided discovery of a potent and orally bioavailable urea-based calcimimetic for the treatment of secondary hyperparathyroidism," Bioorganic \& Medicinal Chemistry Letters, vol. 23, no. 24, pp. 6625-6628, 2013.

[12] U. R. Mane, D. Mohanakrishnan, D. Sahal, P. R. Murumkar, R. Giridhar, and M. R. Yadav, "Synthesis and biological evaluation of some novel pyrido[1,2-a]pyrimidin-4-ones as antimalarial agents," European Journal of Medicinal Chemistry, vol. 79, pp. 422-435, 2014.

[13] V. G. DeVries, J. D. Bloom, M. D. Dutia, A. S. Katocs, and E. E. Largis, "Potential antiatherosclerotic agents. 6. Hypocholesterolemic trisubstituted urea analogs," Journal of Medicinal Chemistry, vol. 32, no. 10, pp. 2318-2325, 1989.

[14] S. L. Nowotarski, B. Pachaiyappan, S. L. Holshouser et al., "Structure-activity study for (bis)ureidopropyl- and (bis) thioureidopropyldiamine LSD1 inhibitors with 3-5-3 and 3-63 carbon backbone architectures," Bioorganic \& Medicinal Chemistry, vol. 23, no. 7, pp. 1601-1612, 2015.

[15] A. Esteves-Souza, K. Pissinate, M. d. Graça Nascimento, N. F. Grynberg, and A. Echevarria, "Synthesis, cytotoxicity, and DNA-topoisomerase inhibitory activity of new asymmetric ureas and thioureas," Bioorganic \& Medicinal Chemistry, vol. 14, no. 2, pp. 492-499, 2006.

[16] J. A. Kowalski, A. D. Swinamer, I. Muegge et al., "Rapid synthesis of an array of trisubstituted urea-based soluble epoxide hydrolase inhibitors facilitated by a novel solid-phase method," Bioorganic \& Medicinal Chemistry Letters, vol. 20, no. 12, pp. 3703-3707, 2010.

[17] S. G. K. Prakash, G. Fogassy, and G. A. Olah, "Microwaveassisted nafion-H catalyzed friedel-crafts type reaction of aromatic aldehydes with arenes: synthesis of triarylmethanes," Catalysis Letters, vol. 138, no. 3-4, pp. 155-159, 2010.

[18] S. Udayakumar, S. Ajaikumar, and A. Pandurangan, "Electrophilic substitution reaction of phenols with aldehydes: enhance the yield of bisphenols by HPA and supported HPA," Catalysis Communications, vol. 8, no. 3, pp. 366-374, 2007.

[19] M. Barbero, S. Cadamuro, S. Dughera, C. Magistris, and P. Venturello, "A new practical synthesis of triaryl and trisindolylmethanes under solvent-free reaction conditions," 
Organic \& Biomolecular Chemistry, vol. 9, no. 24, p. 8393, 2011.

[20] Y. Torisawa, T. Nishi, and J.-i. Minamikawa, "Aldehyde bisarylation by metal triflates including bismuth triflate powder," Organic Process Research \& Development, vol. 5, no. 1, pp. 84-88, 2001.

[21] Z. Hussain, D. Danner, A. Masutani et al., "Effect of long flexible chain reactive monomers on the operating voltage of optically isotropic blue phase liquid crystals," Liquid Crystals, vol. 39, no. 2, pp. 221-230, 2012.

[22] T. Kolasa, D. E. Gunn, P. Bhatia et al., "Symmetrical bis(heteroarylmethoxyphenyl)alkylcarboxylic acids as inhibitors of leukotriene biosynthesis," Journal of Medicinal Chemistry, vol. 43, no. 17, pp. 3322-3334, 2000.

[23] A. R. Burilov, A. S. Gazizov, M. S. Khakimov, N. I. Kharitonova, M. A. Pudovik, and A. I. Konovalov, "Reaction of 1-(2,2dimethoxyethyl)-1-methyl-3-phenylurea with pyrogallol," Russian Journal of General Chemistry, vol. 78, no. 12, pp. 2411-2412, 2008.

[24] A. S. Gazizov, A. V. Smolobochkin, A. R. Burilov, and M. A. Pudovik, "Interaction of 2-naphthol with $\gamma$-ureidoacetals. A new method for the synthesis of 2-arylpyrrolidines," Chemistry of Heterocyclic Compounds, vol. 50, no. 5, pp. 707-714, 2014.

[25] A. S. Gazizov, A. V. Smolobochkin, J. K. Voronina, A. R. Burilov, and M. A. Pudovik, "Acid-catalyzed reaction of (4,4-diethoxybutyl)ureas with phenols as a novel approach to the synthesis of $\alpha$-arylpyrrolidines," Synthetic Communications, vol. 45, no. 10, pp. 1215-1221, 2015.

[26] A. V. Smolobochkin, A. S. Gazizov, J. K. Voronina, A. R. Burilov, and M. A. Pudovik, "Cyclization of 1-(4,4diethoxybutyl)-3-arylureas: a case study," Monatshefte für Chemie - Chemical Monthly, vol. 149, no. 3, pp. 535-541, 2018.

[27] A. S. Gazizov, A. V. V. Smolobochkin, J. K. Voronina, A. R. Burilov, and M. A. Pudovik, "Acid-catalyzed ring opening in 2-(2-hydroxynaphthalene-1-yl)-pyrrolidine-1-carboxamides: formation of dibenzoxanthenes, diarylmethanes, and calixarenes," Tetrahedron, vol. 71, no. 3, pp. 445-450, 2015.

[28] A. V. Smolobochkin, A. S. Gazizov, A. R. Burilov, and M. A. Pudovik, "Synthesis of functionalized diarylbutane derivatives by the reaction of 2-methylresorcinol with $\gamma$-ureidoacetals," Russian Journal of General Chemistry, vol. 85, no. 7, pp. 1779-1782, 2015.

[29] Y. Hayashi, "Pot economy and one-pot synthesis," Chemical Science, vol. 7, no. 2, pp. 866-880, 2016.

[30] F. D. King and S. Caddick, "Triflic acid-mediated phenylation of $\mathrm{N}$-acylaminoalkyl diethylacetals and $\mathrm{N}$-acyl-2-phenyl cyclic amides," Organic \& Biomolecular Chemistry, vol. 9, no. 11, p. 4361, 2011.

[31] J. Y. Kim, D. S. Choi, and M. Y. Jung, "Antiphoto-oxidative activity of sesamol in methylene blue- and chlorophyllsensitized photo-oxidation of oil," Journal of Agricultural and Food Chemistry, vol. 51, no. 11, pp. 3460-3465, 2003.

[32] Y. Fukuda, M. Nagata, T. Osawa, and M. Namiki, "Contribution of lignan analogues to antioxidative activity of refined unroasted sesame seed oil," Journal of the American Oil Chemists' Society, vol. 63, no. 8, pp. 1027-1031, 1986.

[33] A. J. Wagstaff, S. M. Cheer, A. J. Matheson, D. Ormrod, and K. L. Goa, "Paroxetine," Drugs, vol. 62, no. 4, pp. 655-703, 2002.

[34] P. Lotke, "Paroxetine controlled release was effective and tolerable for treating menopausal hot flash symptoms in women," Evidence-Based Medicine, vol. 9, no. 1, p. 23, 2004.
[35] M. Fava, J. Amsterdam, J. Deltito, C. Salzman, M. Schwaller, and D. Dunner, "A double-blind study of paroxetine, fluoxetine, and placebo in outpatients with major depression," Annals of Clinical Psychiatry, vol. 10, no. 4, pp. 145-150, 1998.

[36] H.-J. Liang, F.-M. Suk, C.-K. Wang et al., "Osthole, a potential antidiabetic agent, alleviates hyperglycemia in $\mathrm{db} / \mathrm{db}$ mice," Chemico-Biological Interactions, vol. 181, no. 3, pp. 309-315, 2009.

[37] M.-Z. Zhang, R.-R. Zhang, J.-Q. Wang et al., "Microwaveassisted synthesis and antifungal activity of novel fused Osthole derivatives," European Journal of Medicinal Chemistry, vol. 124, pp. 10-16, 2016.

[38] Z.-C. Wang, Y.-J. Qin, P.-F. Wang et al., "Sulfonamides containing coumarin moieties selectively and potently inhibit carbonic anhydrases II and IX: design, synthesis, inhibitory activity and 3D-QSAR analysis," European Journal of Medicinal Chemistry, vol. 66, pp. 1-11, 2013.

[39] J. A. Kumar, G. Saidachary, G. Mallesham et al., "Synthesis, anticancer activity and photophysical properties of novel substituted 2-oxo-2H-chromenylpyrazolecarboxylates," European Journal of Medicinal Chemistry, vol. 65, pp. 389-402, 2013.

[40] S. Sheshmani, "Catalytic application of two novel sandwichtype polyoxometalates in synthesis of 14 -substituted-14Hdibenzo[a, j]xanthenes," Journal of Chemical Sciences, vol. 125 , no. 2 , pp. $345-351,2013$.

[41] A. V. Smolobochkin, A. S. Gazizov, A. R. Burilov, and M. A. Pudovik, "Reaction of N-cyclohexyl-2-(2hydroxynaphthalen-1-yl)pyrrolidine-1-carboxamide with resorcinol and its derivatives and synthesis of polyphenols," Russian Chemical Bulletin, vol. 65, no. 5, pp. 1377-1379, 2016. 

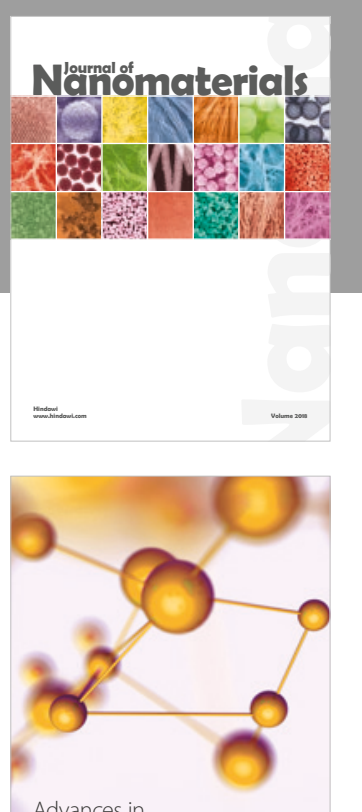

Physical Chemistry
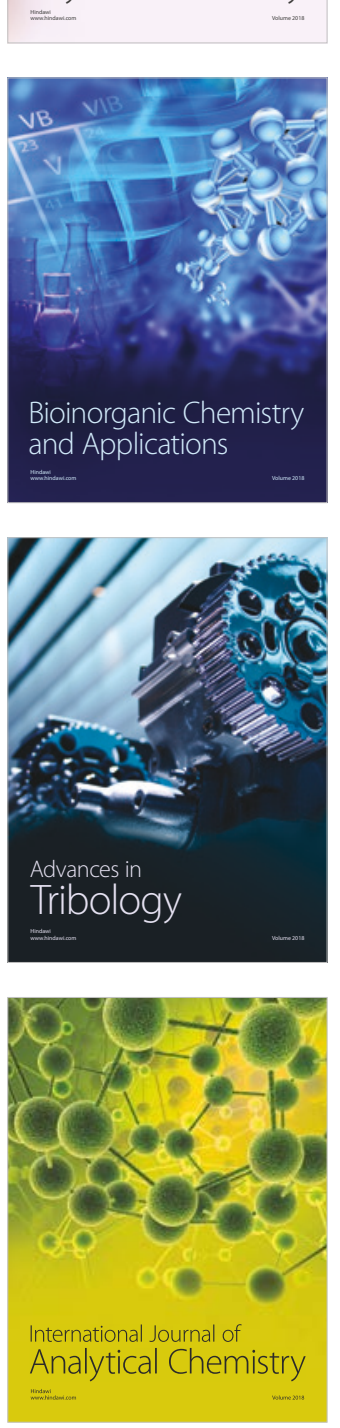

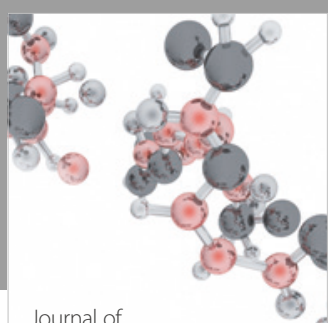

Analytical Methods

in Chemistry

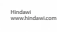

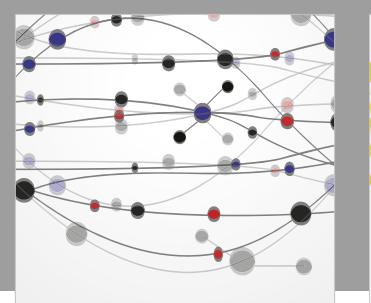

The Scientific World Journal

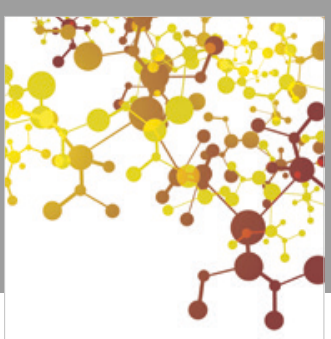

Journal of

Applied Chemistry
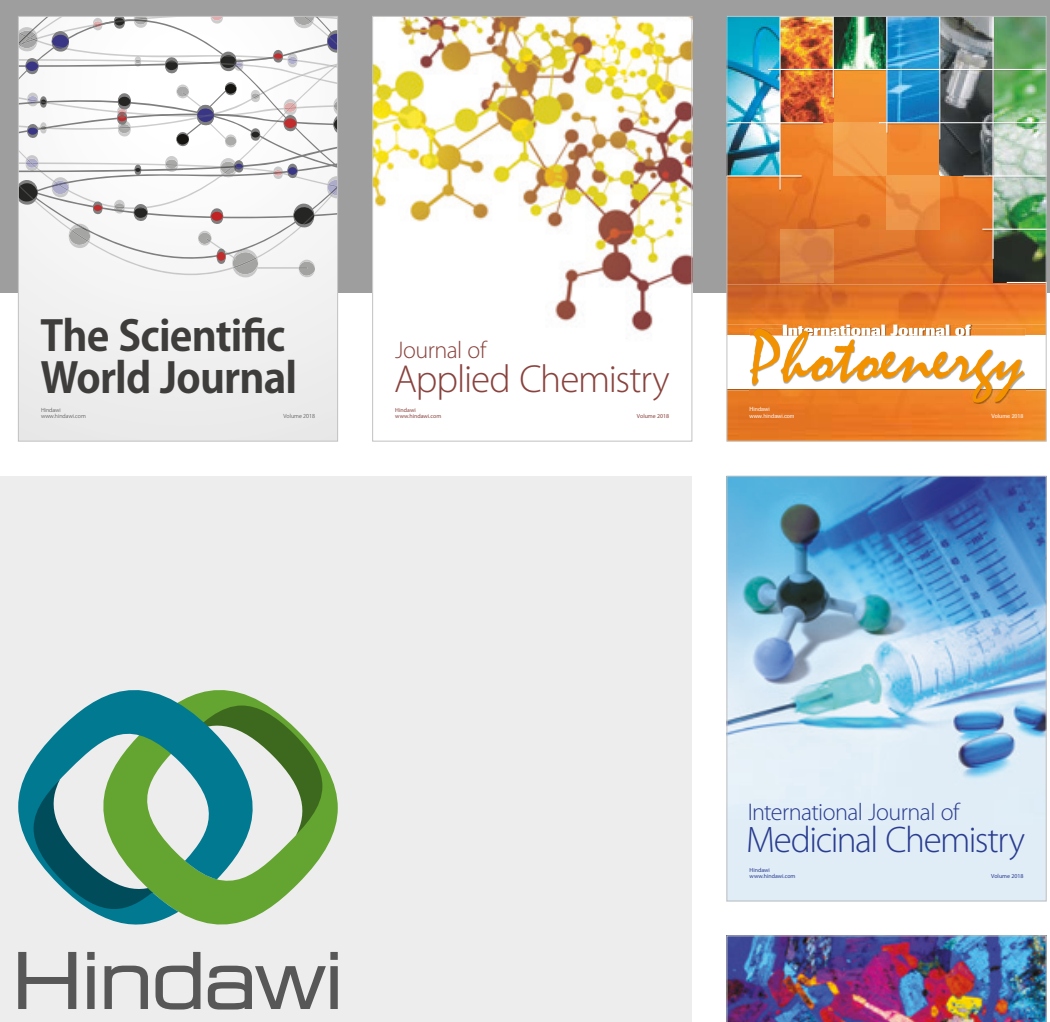

Submit your manuscripts at

www.hindawi.com
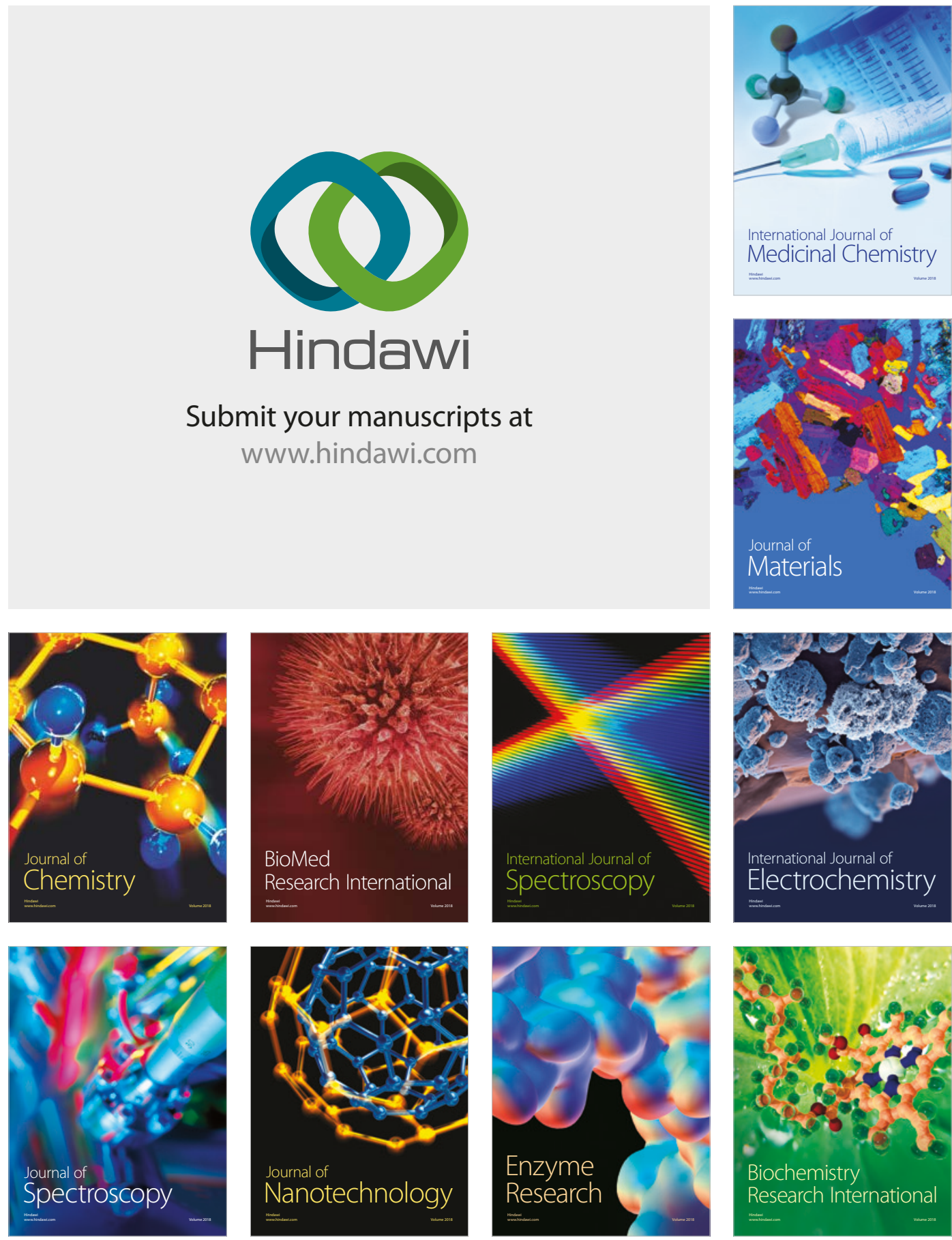
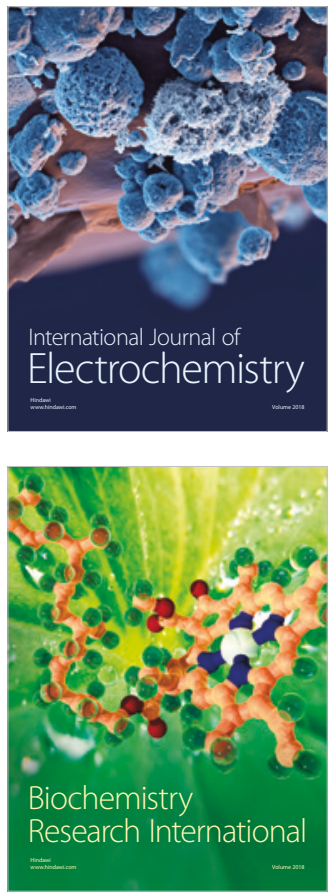